\title{
$X$.
}

\section{Labyrinthbefund eines Falles von Taubheit bei Lenkämie.}

\author{
Von \\ Prof. Dr. Wagenhăuser \\ in Tũbingen.
}

(Von der Redaction übernommen am 26. October 1892.)

So gering an Zahl die klinisehen Beobachtungen über die Erkrankung des Gehörorgans bei Leukämie bisher sind (Gradenigo ${ }^{1}$ ) hat in seiner werthvollen Bearbeitung dieses Themas die betreffenden Beobachtungen zusammengestellt), so spärlich sind auch bis jetzt die Untersuchungen der anatomischen Veränderungen, welche derselben za Grunde liegen. Vier Untersuchungen sind es im Ganzen, die die Literatur in dieser Bezichung aufzuweisen hat: ein Fall von Politzer ${ }^{2}$, der von Gradenigo in der schon oben erwähnten Arbeit, und zwei weitere Fälle von Stein brüg ge. ${ }^{3}$ )

Ich bin in der Lage, mit dem Folgenden eine weitere Beobachtung anreiben zu können, und verdanke es der Liebenswürdigkeit der Herren Prof. v. Lie bermeister und Ba umgarten, welche mir das klinische und anatomische Material dazu überlassen haben.

Leider hat intra vitam eine Ohruntersuchung nicht stattgefunden, ebenso sind die anamnestischen Daten in Bezug auf das Gehörorgan nur mangelhaft; immerhin jedoch glaube ich, dass die Mittheilung des Befundes von Interesse ist.

Der Fall betrifft eine 35 jäbrige Fabrikarbeiterin, Caroline 0 . aus Horkheim, Oberamt Heilbronn, welche am 27. Mai 1891 auf der medicinischen Klinik dahier gestorben ist.

1) Das Gehörorgan bei der Leukämie. Archiv f. Ohrenheilk. Bd. XXIII. S. 242 .

2) Congress zu Basel. 1884, Comptes rendus. p. 132.

3) Zeitschr. f. Ohrenheilk. Bd. XVI. S. 238, und Pathol. Anatomie des Gehörorgans. S. 115 . 
Nach Aussage der die Kranke hierher verbringenden Person (Patientin war bei der Aufnahme auf die Klinik [21. Mai 1891] schon taub, und konnte man sich nur schriftlich mit ihr verständigen) war dieselbe gesund bis April 1889, zn welcher Zeit ihre Erkrankung mit Erscheinungen von Seiten des Unterleibes begonnen hatte. Eine bedeutende Verschlimmerung soll sich eingestellt haben, nachdem Patientin im Herbste 1890 eine Kaltwassercur bei einem Curpfuscher durchgemacht hatte.

Der Arzt, der die Patientin nachher in Behandlnng bekommen und später ihre Ueberführung auf die Klinik veranlasst batte, berichtet über den damaligen Zustand das Folgende: „Nach ihrer Ruickkunft von dort (Kaltwassercur) stellten sich zu Anfang October unter Fieber mittleren Grades geschwulstartige Infiltrationen ron ziemlicher Ausdehnung am rechten Oberarm und rechten Oberschenkel ein und zugleich Verlust des Gehörs auf beiden Seiten." Die Anschwellungen sollen bis gegen Ende des Jahres wieder zurückgegangen sein, die Taubheit aber blieb bestehen. Weiter habe die Kranke vor ihrer Aufnahme an häufigem Erbrechen, Nasenbluten und starken Kopfschmerz gelitten.

Bei der Aufnahme auf die Klinik befand sich Patientin in einem Zustand grösster Abmagerung. Die Untersuchung ergab grossen Milztumor. Brettharte Anschwellung des rechten Oberschenkels und der Glutealgegend rechts. Bedeutende Vermehrung der weissen Blutkörperchen.

In den nächsten Tagen viel Klagen über Kopfschmerz, bäufiges Nasenbluten, das nur auf Tamponade steht. Anch in der linken Glutealgegend kommt eine harte, ödematöse Anschwellung zur Entwicklung.

Am Morgen des 27. Mai plötzlicher Collaps und Tod.

Klinisehe Diagnose: Lineale Leukämie. Grosser. Milztumor. Thrombose der Vena iliaca communis dextra et sinistra.

Die Section ergab: Leukämie. Blut von undurchsichtiger, opaker Beschaffenheit und eigenthümlich mattrother Farbe. Milzvergrösserung. Leber leukämisch infiltrirt. Vergrösserung der retroperitonealen Lymphdrüsen. Knochenmark in leukämischer Hyperplasie. An Stelle der angenommenen Thrombose fanden sich als Ursache der Ansehwellungen ausgedehnte Blutergïsse in die Muskelsubstanz (Hämatome) vor.

Von den beiden der Leiche entnommenen Schläfenbeinen 
wurden äusseres und mittleres Ohr sofort makroskopisch untersucht, wobei sich völlig normaler Befund ergab. Nur an der Labyrinthwand der Paukenhöhle einzelne erweiterte Gefässe und Gefässästchen. Nirgends eine Spur von Exsudat in den Hohlräumen des Mittelohres.

Die beiden das Labyrinth umsehliessenden Felsentheile wurden zum Zwecke der späteren histologisehen Untersuchung in Müller'sche Flüssigkeit eingelegt. Entkalkung in 5 proc. Salpetersäure, Celloidineinbettung, Pikrocarminfärbung.

Der Bearbeitung der Präparate hatten sich in beiden Felsenbeinen grosse Schwierigkeiten entgegengestellt. Alsbald nach den ersten Frontalschnitten durch die Schnecke stiess das Messer auf einen spitzen, harten Knochenkern, nach hinten von der Schnecke gelegen, der eine weitere Schnittführung daselbst unmöglich machte. Das gleiche Hinderniss bot sich dar beim Versuche, vom Warzenfortsatz her zu schneiden. Nochmaliges Einlegen in verstärkte (bis zu 20 Proc.) Entkalkungsflüssigkeit bewirkte nur langsame und oberflächliche Erweichung der betreffenden Partien, so dass ich schliesslich den Versuch unternahm, die Pyramide durch einen senkrecht auf die Längsaxe derselben gerichteten Schnitt in zwei Hälften zu zerlegen. Auch hierbei kam das Messer wieder in der Mitte des Präparates auf einen harten Kern, der alsdann, nach kreisförmiger Durchtrennung der entkalkten Partien, mit einem feinen Meissel durchschlagen wurde.

Die auf diese Weise erhaltenen Flächen zeigten, medialwärts von den Räumen des Labyrinths gelegen, einen länglich-runden, $31 / 2 \mathrm{Mm}$. breiten Knochenkern von völlig elfenbeinharter Beschaffenheit, der offenbar, nach vorn und hinten sich verschmälernd, die ganze Länge der Pyramide durchsetzte. Wiederholte Entkalkungsversuche lieferten auch jetzt nur so unbefriedigende Ergebnisse, dass ich mich entschloss, den harten Knochenkern aus den Präparaten herauszulösen. Wenn auch auf diese Weise die Continuität der Schnitte gestört war, so liessen sich doch immer noch für die Beurtheilung der Verbältnisse verwerthbare Einzelbilder erlangen. (Ein ähnlicher Befund von harter Knochenmasse in einem sonst völlig entkalkten Präparate ist mir neuerdings wieder begegnet. Da es für mich weniger Werth besitzt, will ich es geduldig weiteren Entkalkungsproceduren unterwerfen und bin begierig, ob sie zu besseren Resultaten führen.) 


\section{Histologischer Befund des inneren Ohres.}

Schnecke.

Am stärksten ausgeprägt, und zwar fast ganz gleichmässig in beiden Ohren, fanden sich die pathologischen Veränderungen in der oberen und mittleren Windung der Schnecke. Daselbst erscheint die Scala vestibuli fast völlig ausgefiullt von einem Extravasate, das an einzelnen Schnitten deutlich seine Zusammensetzung aus rothen und farblosen Blutkörperchen erkennen lässt. In der unteren Windung enthält die Vorhofstreppe nur einen schmalen, der Lamina spiralis ossea aufliegenden Saum körniger Massen und darüber, theils einzeln, theils in Gruppen von 8-10 Stiick angeordnet, grosse rundliche Zellen mit körnigem Inhalte.

In der Scala tympani sämmtlicher Windungen zeigt sich an der basalen Umrandung der gleiche Saum körniger Massen und die Anwesenheit der vorgenannten grossen Zellen.

Im Ductus cochlearis allerorten körnige Massen, hier und da noch Zellcontouren erkennen lassend, die Membrana Corti und das Corti'sche Organ umhüllend und stellenweise völlig verdeckend. Zellen des Corti'schen Organs und Membrana Reissneri meist sehr gut erhalten.

Im Stamme des Hörnerven kein Extravasat nachzuweisen, wohl aber im Canalis ganglionaris der mittleren Windung. Frei erscheint ferner der Nerv. facialis. Gefässe des Modiolus stark geschlängelt und strotzend gefüllt. Auch sonst auf den Durchschnitten der Windungen überall starke Gefässfüllung erkennbar. Vom Aquaeductus cochleae und runden Fenster keine Präparate erhalten.

\section{Vorhof.}

Von den Vorhof'sgebilden waren nur wenige brauchbare Bilder zu erlangen. Im Utriculus grosses Extravasat, theils zerfallen, theils noch Zellcontouren enthaltend. Auch hier zahlreiche der oben beschriebenen grossen rundlichen Zellen. Periostale Auskleidung des Vestibulums an der medialen Wand stark verdickt. Zwischen ihr und der Wand des Utriculus neugebildetes Bindegewebe mit spärlichen Rundzellen und bald lockerer, bald dichterer Gewebsanordnung. In der Cisterna perilymphatica massenhaftes Extravasat, ebenso auch in der Ampulla inferior. In den übrigen Ampullen nur spärliche Extravasation, dagegen mäehtige vom Periost ausgehende Bindegewebsentwick- 
lnng. Macula utriculi und Cristae ampullares mit relativ gut erhaltenem Epithel. Kleine Extravasate in den entsprechenden Nervenzweigen.

$$
\text { Bogengänge. }
$$

Durchschnitte der Bogengänge liessen erkennen, dass hier und wiederum ganz gleichmässig anf beiden Seiten - die Veränderungen am weitesten zur Entwicklung gekommen waren.

Der perilymphatischo Raum erscheint ron Bindegew eb e ausgefüllt, mit bald mehr, bald weniger dichter Anordnung, stellenweise ungemein zellenreich und von neugebildeten Gefässen durchsetzt. An anderen Durchschnitten zeigt sich neugebildete Knochenmasse als unregelmässig zackige, von der Wandung ausgehende Vorsprïnge, oder als Erfüllung des ganzen Raumes durch Knochen.

Der häutige Kanal ist überall erhalten geblieben als rundlicher oder anf einzelnen Bildern durch die Knochenmasse winklig oder nierenförmig eingebogener Gang. Einzelne Durchschnitte enthalten kleine Extravasate, während andere leer erscheinen.

\section{Epikrise.}

Wenn es auch im Interesse der völligen Würdigung des Falles zu bedauern ist, dass genauere Angaben uber die Entwicklung der Ohraffection, das Vorhandensein oder Fehlen von Schwindel und Ohrgeränschen, sowie über functionelle Prüfungen nicht vorliegen, so bietet derselbe immerhin für die Beurtheilnng der Obrcomplication bei Lenkämie verwerthbare Aufschlüsse.

Es bandelte sich um ein Individunm, bei dem es im Laufe der Erkrankung vielfach zu Blutungen gekommen war (die Anschwellangen am rechten Oberarm und Oberschenkel, welche gleichzeitig mit der Ohraffection in Erscheinung getreten sind, dürfen wohl auch auf Blutergïsse zurückgeführt werden). Dieser ausgesprochenen Neigung za Blutungen entspricht auch der Befund von Hämorrhagien in beiden Labyrinthen.

Halten wir uns zunächst an diesen Befund und vergleichen wir ihn mit den Ergebnissen der früheren Untersuchungen, so finden wir ihn in Uebereinstimmung mit denselben.

In den vier vorliegenden Fällen wurden Extravasate im Bereiche des Gehörorgans nachgewiesen. Anf die Paukenhöhle beschränkt im Falle von Gradenigo, im Mittelohre und Labyrinth bei Steinbrügge and schliesslich nur im Labyrinth in dem weiteren Falle von Steinbrïgge und dem von Politzer. Im 
Politzer'schen Falle handelte es sich mehr um Extravasate aus Lymphzellen bestehend, während in den übrigen Befunden rothe Blutkörperchen vorgeherrscht haben.

Als weiter ubereinstimmend mit den bisherigen Ergebnissen ist sodann die nachgewiesene Bindegewebs- and Knochenneubildung im Labyrinth zu bezeichnen.

Politzer berichtet ron ausgedehnter Bindegewebs" und Knochenneubildung in der Schnecke und bindegewebiger Erfüllung der Räume der knöchernen Bogengänge. Die erste Mitheilung von Stein brüg g e weist Knochenneubildang in den Bogengängen auf, im Falle ron Gradenigo fanden sich bindegewebige Neubildungen in den oberen Partien der Trommelhöhle vor, und nur die letzte Mittheilnng von Steinbrügge, bei welcher die Affection des Ohres offenbar jüngeren Datums war, hat nichts davon aufzuweisen.

Unser Befund bildet also in jeder Hinsicht eine Bestätigung der Resultate früherer Untersuchungen, und darin liegt zunächst sein Werth. Eine weitere Bedeutung ditrfte ihm vielleicht noch zukommen in Bezug auf die Frage nach der Entwicklung der erwähnten Neubildungsproducte.

In den drei früheren Fällen, bei welchen derartige Befunde nachgewiesen waren, hatten chronische, theilweise eitrige Processe des Ohres vorher schon bestanden, ausserdem war bei zweien derselben eine syphilitisehe Infection vorhanden gewesen. Auf diese Entzündungsvorgänge fübren nun Gradenig o sowohl, wie anch Stein brügge die Bindegewebs- und Knochenneubildungen zuriek. Sie sind geneigt, dieselben als Residuen vorausgegangener Entzündung anzusehen, zu welchen dann erst später die leukämische Erkrankung des Obres hinzugekommen sei. Der erstgenannte Autor hebt das Vorausgegangensein oder die gleichzeitige Existenz eines entzündlichen Processes im Obr als wesentlichen prädisponirenden Factor der Ohreomplication bei Leukämie geradezu hervor.

Von Syphilis war in unserem Falle nichts bekannt. Auch für die Annahme vorausgegangener entzündlicher Vorgänge lag kein beweiskräftiges Moment vor, so dass also eine derartige Erklärung für unseren Fall keine Geltung besitzt. Aber auch ohne Zuhülfenahme derselben lässt sich der Befund, wie ich glaube, erklären.

Nach meiner Ansicht bildete die Hämorrhagie ins Labyrinth, wabrscheinlich in das Bereich der Bogengänge und in den Vorhof, 
das Primäre und zugleich das, was direct mit der Leukämie in Zusammenhang gebracht werden kann. Durch die Blutung wurde ein entziundlicher Vorgang in der Nachbarschaft der afficirten Partie hervorgerufen, als dessen Endprocess die betreffenden Bindegewebs- und Knochenneubildungen anzusehen wären.

In dem Letzteren liegt nichts, was einer besonderen Begrïndung bedürfte, denn derartige Befunde wurden bisher häufig als Product der verschiedenartigsten Entzündungsprocesse des Labyrinths nachgewiesen. Die Frage ist nur die: Lässt sich eine Hämorrhagie in das Labyrinth als Ursache einer Entzündung daselbst aufstellen?

Beweise dafür sowohl, wie auch dagegen scheinen nicht vorhanden zu sein. Steinbrü gig $\mathrm{e}^{1}$ ) lässt es zweifelhaft, ob Blutextravasate in das Labyrinth (nur grössere haben hier in Betracht zu kommen) an und für sich, ohne Hinzutreten organischer Entzündungserreger oder toxischer Substanzen, eine Entzündung der Gewebe daselbst hervorrufen können. Ich möchte es annehmen, mich stïtzend auf die Beobachtungen der allgemeinen Pathologie, denn nach Ziegler ${ }^{2}$ ) ist die Anwesenheit geronnener Exsudate sowohl, als auch geronnenen Blutes in Geweben stets von Entzündungsprocessen gefolgt, welche indessen bei Abwesenheit von Verunreinignngen niemals sehr heftige werden.

Die Annahme einer Entzündung des Labyrinths infolge der Blutung hätte also danach nichts Unwahrscheinliches, ebensowenig auch die weitere Annahme, dass durch wiederbolte Blutungen der entzïndliche Vorgang daselbst unterhalten und za einer solchen Intensität gesteigert worden ist, dass er schliesslich zum beschriebenen Endprocess geführt hat. $\mathrm{Ob}$ und wie weit diese Folgerungen richtig sind, werden spätere Untersuchungen lehren.

1) Pathologische Anatomie des Ohres. S. 86 .

2) Lehrbuch der pathologischen Anatomie. 1892. Bd. I. S. 266. 